\title{
Caracterizando las Necesidades de las Pymes para Implementar Mejoras de Procesos Software: Una Comparativa entre la Teoría y la Realidad
}

\author{
Mirna Muñoz ${ }^{1}$, Gloria Gasca ${ }^{2}$, Claudia Valtierra ${ }^{1}$ \\ \{mirna.munoz, claudia.valtierra\}@cimat.mx, gpgasca@udem.edu.co \\ ${ }^{1}$ Centro de Investigación en Matemáticas- Unidad zacatecas, Av. Universidad No. 222, 98068, Zacatecas, \\ México \\ 2 Universidad de Medellín, Medellín, Colombia
}

DOI: 10.4304/risti.e1.1-15

\begin{abstract}
Resumen: Actualmente en el ámbito del desarrollo de software a nivel mundial las pymes ocupan un considerable porcentaje del número de empresas generando el $52 \%$ del producto interno bruto de los países y alrededor del $72 \%$ de los empleados formales. Sin embargo, aun cuando es importante asegurar la calidad en sus productos, existe una carencia en la implementación de procesos o modelos formales enfocados en el desarrollo de software que las ayuden a las organizaciones a dirigir esta necesidad. Este artículo muestra una comparativa entre teoría y realidad de la caracterización de necesidades que presentan las pymes para implementar una mejora de procesos de software exitosa. En específico el estudio presentado en este artículo se centra en la Región de Zacatecas, México y los resultados obtenidos al realizar una revisión de literatura enfocada en la caracterización de pymes.
\end{abstract}

Palabras-clave: Pymes; mejora de procesos de software; necesidades de SPI en Pymes; modelos y estándares de calidad; revisión literaria.

Characterizing SME's needs for implementing a software process improvement: A comparative between the reality and the theory

\begin{abstract}
Nowadays in the software development industry SMEs occupy a considerable percentage of the number of companies generating the $52 \%$ of the gross domestic product of the countries and around $72 \%$ of the formal employees. However, even when guarantee the software quality is important for SMEs, they have a lack in the implementation of software development formal processes that help them to address this need. This paper shows a comparative between reality and theory focused on the characterization of the needs that SMEs have to face when implementing a software process improvement. The paper is focused on showing the results of analyzing SMEs from Zacatecas Region of Mexico and the results obtained performing a systematic review results and the SME's from Zacatecas Region results are included.
\end{abstract}


Keywords: SMEs; software process improvement; SPI SMEs' needs; quality models and standards, systematic review.

\section{Introducción}

En la actualidad las pequeñas y medianas empresas de software se consideran una pieza importante en la economía mundial de la industria de Software. En las últimas décadas esta industria ha crecido fortaleciéndose al máximo, por lo que representan una de las grandes actividades de desarrollo para la producción de empleos (Moreno, 2008) (Ministerio de Industria, Energía y Turismo, 2013).

En este trabajo de investigación se aborda el tema de caracterizar las pymes de desarrollo de software de tal forma que se puedan establecer las necesidades que éstas deben cubrir para que se logre una implementación de mejora de procesos exitosa en la que se enfoque a la organización y sus objetivos del negocio.

Para lograr esta caracterización, se realizó una revisión de literatura, que permitió la obtención de información, mediante la utilización de un método formal de investigación (Kitchenham \& Charters, 2007). Además, para fortalecer esta caracterización se realizó una investigación de campo en la región de Zacatecas, con lo cual se ha podido realizar una comparativa entre los resultados obtenidos.

El objetivo de este artículo es presentar una caracterización de las pymes desarrolladoras de software para identificar las necesidades que éstas deben cubrir para implementar una iniciativa de mejora en sus procesos de software. El artículo está estructurado como sigue: en la sección 2 se presenta una breve descripción de las empresas contempladas bajo el concepto de pymes; en la sección 3 se presenta un resumen de la revisión de literatura realizada; en la sección 4 se presentan los resultados de la revisión de literatura enfocado en: los modelos y estándares más utilizados, la mejora de procesos, las principales limitaciones que presentan las pymes para implementar la mejora de procesos software (SPI, Software Process Improvement) y las ventajas de implementación de SPI en las Pymes; en la sección 5 se presenta una investigación de campo que se realizó en el estado de Zacatecas; en la sección 6 se presenta una comparativa de los resultados obtenidos de la caracterización de la pymes mediante la revisión de literatura y las pymes de la región de Zacatecas y; finalmente en la sección 7 se presentan las conclusiones y trabajo futuro.

\section{Pymes}

El término "pymes" es utilizado al clasificar las empresas de acuerdo al número de trabajadores, sin embargo hay que tener en cuenta que este número varía de acuerdo a la región o país en la cual la pyme se establezca (Hogan, Smith, \& Thomas, 2002), por ejemplo, en Irlanda casi el $99 \%$ de las pymes son pequeñas y emplean menos de 50 personas (Scott, Jeffery, Carvalho, D’Ambra, \& Rutherford, 2001), en Australia el 98\% de las empresas pequeñas y medianas tiene menos de 20 empleados y en México existen aproximadamente 4 millones 15 mil empresas de las cuales 99.8\% son Pymes con un rango de entre 1 y 130 empleados (Secretaria de Economía, Pymes, 2013). 
En base a lo antes mencionado, una categorización que puede ser tomada como genérica para las pymes se muestra en la Tabla 1 (Ministerio de Industria, Energía y Turismo, 2013).

Tabla 1 -Categorías de Pymes Desarrolladoras de Software

\begin{tabular}{cc}
\hline Categoría de la pyme & Número de personas \\
\hline Mediana & 51 a 130 \\
\hline Pequeña & 10 a 50 \\
\hline Micro & 1 a 9 \\
\hline
\end{tabular}

Algunas de las ventajas de las pymes son los siguientes (Secretaria de Economía, Pymes, 2013): 1) Son un importante motor de desarrollo del país contribuyendo al desarrollo local y regional; 2) Por su dinamismo tienen posibilidad de crecimiento y de llegar a convertirse en una empresa grande; 3) Absorben una porción importante de la población económicamente activa, debido a su gran capacidad de generar empleos y; 4) Asimilan y adaptan nuevas tecnologías con relativa facilidad.

\section{Revisión de Literatura}

Con la finalidad de obtener la situación actual sobre el entorno de las pymes desarrolladoras de software, se ha llevado a cabo la revisión de la literatura utilizando un protocolo de revisión sistemática.

La revisión de literatura es un método que permite identificar, evaluar e interpretar investigaciones con respecto a un tema específico. El objetivo de emplearla es la obtención de información relevante de un tema mediante la utilización de un método formal (Kitchenham \& Charters, 2007).

Como primer paso para la realización de la revisión de literatura se realizaron las siguientes preguntas de investigación que permiten caracterizar la mejora de procesos de acuerdo al tipo de empresa en la que se implemente la mejora (Valtierra, Muñoz \& Mejia, 2013). Las preguntas de investigación se establecerán de la siguiente manera: (a) ¿En qué tipo de dominio se encuentra la pyme de desarrollo de software?; (b) ¿Con cuántos empleados está organizada la pyme?; (c) ¿Qué procesos manejan en la pyme y sí se ha tenido alguna experiencia para la implementación de iniciativas de mejora de procesos de software? y (d) ¿Qué problemas han tenido en la implementación de mejora de proceso?

Dichas preguntas proporcionan información sobre las características de las pymes de cara a la implementación de mejora, por lo que han permitido identificar las necesidades de las pymes para la implementación de mejora de procesos. Para dar respuesta a las preguntas se identificaron las siguientes palabras claves: SMEs (Small and Medium Enterprises), process, software, development, improvement y needs.

Siguiendo el protocolo de revisión de literatura se generaron las cadenas de búsqueda para la obtención de publicaciones de acuerdo al tema abordado en esta investigación. Las cadenas resultantes son las siguientes: 
- (process) and (smes) and (software or development).

- (sme) and (software process improvement) and (needs).

Las fuentes seleccionadas para ejecución de la revisión de literatura fueron: IEEE, ACM, SEI y Springer Link, cabe resaltar que las cadenas fueron adecuadas a cada buscador específico de cada fuente. Finalmente, ejecutando las cadenas de búsqueda en las 4 fuentes seleccionadas se obtuvieron 6441 resultados.

Posteriormente, se aplicaron los criterios de inclusión y exclusión (véase la Tabla 2) con el fin de filtrar los resultados; de lo anterior se obtuvo una lista de artículos relevantes de los cuales se realizó una revisión más profunda de su contenido desde el resumen hasta las conclusiones, obteniendo finalmente 40 estudios primarios, como base para la investigación presentada en este artículo. Cabe resaltar que esta revisión está constituida principalmente por artículos de congresos internacionales; aunque no se descartaron algunos artículos de revistas, JCRs y, reportes técnicos relevantes para la investigación. En la siguiente sección se muestra el análisis de los principales resultados obtenidos.

Tabla 2 -Criterio de inclusión y exclusión

Criterios de inclusión

Estudios con idiomas inglés o español.

- $\quad$ Estudios dentro del periodo comprendido desde el año 2000 hasta la fecha.

- $\quad$ Estudios que contengan en el título las palabras claves.

- $\quad$ Estudios que en el resumen contenga información sobre la mejora de procesos de software.

- $\quad$ Estudios con 4 hojas o más.

- Estudios que contengan información sobre la mejora de procesos de software en pymes desarrolladoras de software.

- $\quad$ Estudios que sean de congresos internacionales, revistas indexadas y JCRs.

\section{Criterios de exclusión}

Estudios que no sean de los idiomas indicados.

- $\quad$ Estudios que no estén dentro del periodo indicado.

- $\quad$ Estudios repetidos en más de una fuente y/o cadenas.

- $\quad$ Estudios con menos de 4 hojas.

- Estudios que no contengan información relevante a la mejora de procesos de software en pymes desarrolladoras de software.

\section{Análisis de Resultados}

1.1. En esta sección se incluye el análisis de los resultados obtenidos enfocados en los siguientes aspectos: 1) las pymes analizadas en la revisión de la literatura; 2) modelos y estándares más utilizados por las pymes; 3) los procesos más enfocados al implementar mejoras; 4) las principales limitaciones que tienen las pymes al implementar iniciativas de mejoras de procesos software; y 5) las ventajas que conlleva implementar una mejora de procesos en las pymes. 


\subsection{Pymes analizadas en la revisión de literatura}

De los 40 estudios primarios obtenidos de la revisión sistemática se analizaron 899 pymes. La Tabla 3 muestra un análisis de las pymes extrayendo aquellas que han implementado una iniciativa de SPI.

Tabla 3 -Pymes mediante la Revisión de literatura

\begin{tabular}{lcc}
\hline País & Pymes & No. Empleados \\
\hline Australia & 9 & $4-60$ \\
\hline Irlanda & 30 & $2-129$ \\
\hline Colombia & 16 & $1-20$ \\
\hline México* & 5 & $8-62$ \\
\hline Perú & 3 & 6 \\
\hline Chile & 8 & $3-125$ \\
\hline Francia & 1 & 14 \\
\hline India & 5 & $10-49$ \\
\hline Suecia & 1 & 120 \\
\hline España & 11 & $10-125$ \\
\hline
\end{tabular}

* Las empresas contempladas en esta sección no incluyen las empresas de la región de Zacatecas.

\subsection{Modelos y estándares más utilizados}

Para la implementación de mejora de procesos de software se han identificado que los modelos y estándares más utilizados por las pymes se muestran a continuación:

- Modelos y estándares enfocados en la evaluación de la madurez de los procesos de software: el 67\% hacen referencia a ISO 15504; el 17\% a OWL y finalmente el 16\% a EPA (Ver Figura 1).

- Modelos y estándares enfocados en la mejora de procesos de software: el 25\% hace referencia a CMMI y a ISO 15504, el 19\% a MesoPyme, el 13\% Moprosoft, el 6\% a TSP, el 6\% IDEAL y finalmente el 6\% ISO 9001:2000 (Ver Figura 2).

- Modelos y estándares enfocados en el ciclo de vida del software: el $66 \%$ hace referencia a ISO 12207, el 26\% a RUP y finalmente el 8\% a ISO 29110 (Ver Figura 3).

- Modelos y estándares enfocados en las pymes: el 43\% hace referencia a MesoPyme, el 29\% Moprosoft y finalmente el 14\% a MSP y 14\% ISO/IEC 29110 (Ver Figura 4).

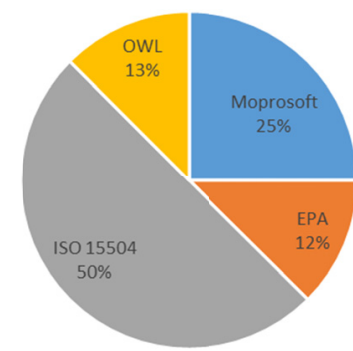

Figura 1 - Modelos y estándares específicos para la evaluación de la madurez de los procesos de software

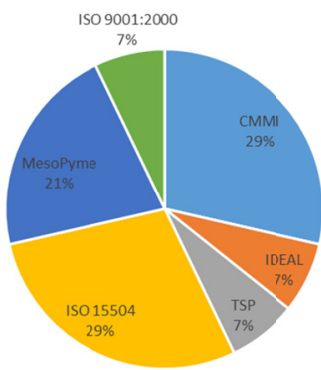

Figura 2 - Modelos y estándares específicos para la mejora de proceso de software 


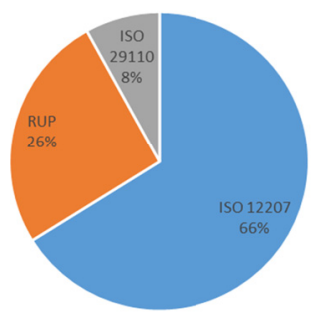

Figura 3 - Modelos y estándares específicos para el ciclo de vida del software

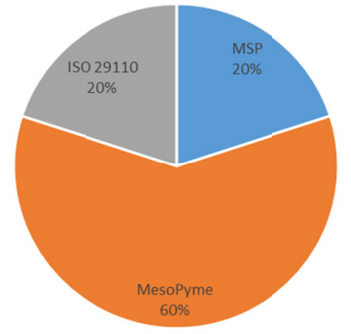

Figura 4 - Modelos y estándares específicos para las Pymes

\subsection{Procesos mejorados}

Los modelos y estándares antes mencionados han sido implementados en las empresas para mejorar sus procesos de software. Los datos arrojados en el análisis muestran que los procesos que son más enfocados para la implementación de mejora, como se observa el 27\% Planificación de Proyectos (PP); el 19\% Gestión de Requisitos (REQM); el 16\% Gestión de Configuración (GM); el 12\% Gestión de Riesgos (RSKM). En porcentaje menores se pueden observar, el 8\% Verificación y Validación (Ver \&Val); el 7\% Desarrollo de Requisitos (RD); el 6\% Aseguramiento de la Calidad de Proceso y del Producto (PPQA) y el 5\% Monitorización y Control del Proyecto (PMC).

\subsection{Principales limitaciones}

Para tener una mejor apreciación de las principales limitaciones, éstas se han clasificado en 6 grupos, esta clasificación se tomó de 2 trabajos previos (Muñoz et al., 2012), (Mas \& Amengual, 2012), las cuales se muestra a continuación:

a) Organización: (1) Son altamente dependientes de los clientes; (2) Desconocen la importancia que tiene el proceso de desarrollo sobre la calidad del producto; (3) No siguen un ciclo de desarrollo de software.

b) Recursos Financieros: (1) Recursos limitados, ya que no cuentan con suficiente capital económico para invertir en mejora de procesos; (2) Dependen de apoyo externo para implementar una mejora de procesos.

c) Recursos Humanos: (1) Carecen de personal, ya que el número de sus empleados suelen ser mínimo; (2) No hay roles definidos, por este motivo los empleados realizan varias funciones; (3) Los empleados carecen de conocimientos sobre los métodos para la mejora de procesos.

d) Procesos: (1) No tienen procesos definidos, por lo que se desarrolla el software de forma artesanal; (2) Es muy costoso implementar procesos y arrojar resultados sobre la implementación de mejora de proceso; (3) Es muy difícil adoptar un Modelo y Estándar para la mejora de procesos para atacar los objetivos y la visión de ésta.

e) Proyectos: (1) Tiende a trabajar proyectos muy pequeños, los cuales conllevan poco tiempo para su realización; (2) La implementación de un proceso toma mucho tiempo; (3) Implementan una mejora de procesos por que los clientes lo exigen. 
f) Modelos y Estándares: (1) Tienen poca o ninguna experiencia en la adopción de modelos y estándares de mejora de procesos software (SPI) y métodos de evaluación; (2) Se tienen que adecuar al modelo y/o estándar que se va a aplicar.

\subsection{Ventajas al implementar mejoras en las pymes}

Esta sección se presenta algunas de las ventajas que se pueden conseguir mediante la aplicación de mejoras de procesos de desarrollo de software para pymes. Las ventajas mostradas están enfocadas en la organización y en los recursos humanos (ver Tabla 4).

\section{Investigación de Campo}

Para realizar la investigación de campo se diseñó una encuesta que sirvió como guía durante las entrevistas a pymes de la Región de Zacatecas, con el fin de obtener las principales características de las empresas y su experiencia con el manejo de procesos o mejora de procesos.

Tabla 4 -Ventajas al implementar mejoras en las pymes

\begin{tabular}{|c|c|}
\hline Ventajas para la organización & Ventajas para los recursos humanos \\
\hline $\begin{array}{l}\text { Lograr resultados medibles en proyectos } \\
\text { rápidamente, con la implementación de varias } \\
\text { iteraciones de los ciclos de mejora en los cuales } \\
\text { ha sido aplicada (Pino, García, \& Piattini, 2007). } \\
\text { Mejorar la calidad del software que es } \\
\text { producido y la productividad con la que se } \\
\text { trabaja (Pino, García \& Piattini, 20o7), (Valdés, } \\
\text { Astudillo, Visconti \& López, 201O). } \\
\text { Agilizar y facilitar la adopción de modelos y } \\
\text { estándares (Valdés, Astudillo, Visconti, \& López, } \\
\text { 201o). } \\
\text { Definir los procesos y estar en constante mejora } \\
\text { (Serrano, de Oca, \& Cedillo, 20o3), (Pino, } \\
\text { García, \& Piattini, 20o9). } \\
\text { Orientar la visión y los objetivos de las pymes } \\
\text { (Serrano, de Oca, \& Cedillo, 20o3), (Pino, } \\
\text { García, \& Piattini, 20o9). } \\
\text { Ser más rentable (Bedini, Llamosa, Pavlovic, \& } \\
\text { Steembecker, 20o5). } \\
\text { Promover el crecimiento al mercado exterior } \\
\text { (Revankar, Mithare, \& Nallagonda, 20o5). } \\
\text { Guiar para la implementación de SPI } \\
\text { (Landaeta, \& Amescua, 20o8). } \\
\text { Reconocimiento Global (Ibrahim \& Ali, 2011). } \\
\text { Aumento de la productividad (Jeners, Clarke, \& } \\
\text { O'Connor, 2013). } \\
\text { Reducción de densidad de defectos (Jeners, } \\
\text { Clarke, \& O'Connor, 2013). }\end{array}$ & 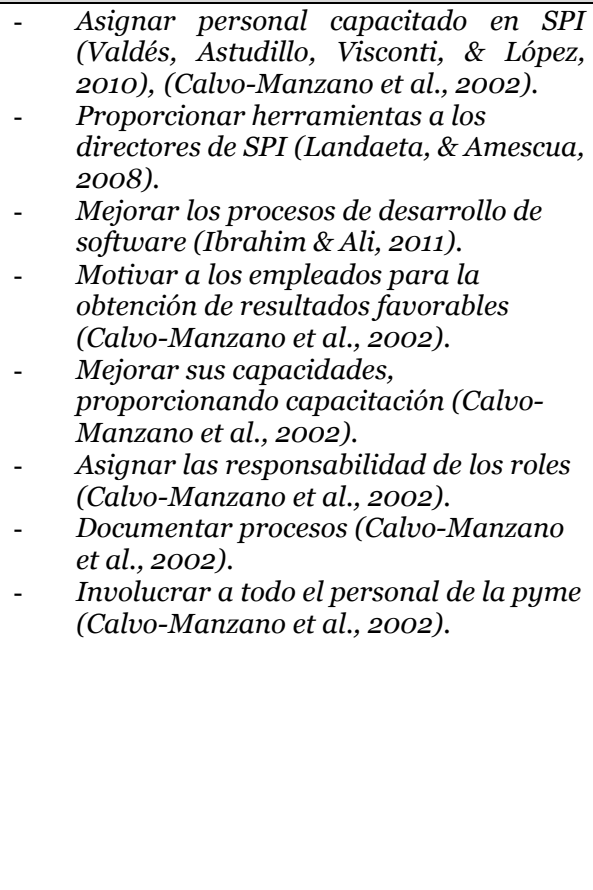 \\
\hline
\end{tabular}

A continuación, se incluye primero la descripción de las pymes analizadas y el cuestionario utilizado y se continua con el análisis de los resultados obtenidos enfocados en los siguientes aspectos: 1) las pymes analizadas en la revisión de la literatura; 2) modelos y estándares más utilizados por las pymes; 3) los procesos más enfocados al implementar mejoras; 4) las principales limitaciones que tienen las pymes 
al implementar iniciativas de mejoras de procesos software; y 5) las ventajas que conlleva implementar una mejora de procesos en las pymes.

\subsection{Pymes analizadas mediante entrevistas}

Se realizó un análisis de la información extraída mediante las entrevistas realizadas en 7 pymes de la región de Zacatecas, con la finalidad de observar el entorno de las pymes, por razones de confiabilidad serán nombradas como pyme1, pyme2, pyme3, pyme4, pyme5, pyme6 y pyme7.

En la Tabla 5 muestra un análisis del número de empleados que tiene cada pyme entrevistada y a continuación se presenta una breve descripción de cada una de las pymes.

Tabla 5 -Pymes Zacatecanas

\begin{tabular}{cc}
\hline Nombre & No. Empleados \\
\hline pyme1 & $\mathrm{n} / \mathrm{a}$ \\
\hline pyme2 & 37 \\
\hline pyme3 & 4 \\
\hline pyme4 & 18 \\
\hline pyme5 & 9 \\
\hline pyme6 & 2 \\
\hline pyme7 & 16 \\
\hline
\end{tabular}

\subsubsection{Descripción de las pymes}

- pyme1 es una empresa dedicada a desarrollar productos y servicios de alto impacto en la educación de niños y adolescentes. En la actualidad no cuentan con personal, sino que contratan personal externo para el desarrollo de un proyecto.

- pyme2 es una empresa dedicada a la consultoría TI, marketing, medios digitales y desarrollo web. En la actualidad cuenta con una plantilla de personal de 37 empleados, entre desarrolladores, diseñadores gráficos, personal de mantenimiento y directivos.

- pymez es una empresa dedicada al desarrollo de software, desarrollo web, soporte y marketing. En la actualidad cuenta con una plantilla de personal de 4 personas, entre desarrolladores y marketing.

- $\quad$ pyme4 es una empresa que pertenece a una institución educativa la cual se dedica al desarrollo de software para dicha institución. En la actualidad cuenta con una plantilla de personal de 18 empleados, entre desarrolladores, líderes de proyectos y administrativos.

- pymes es una empresa dedicada al desarrollo de software de alta calidad. En la actualidad cuenta con una plantilla de personal de 9 empleados, distribuidos en el estado de Zacatecas y en la ciudad de México.

- $\quad$ pyme6 es una empresa que inicio como una empresa desarrolladora de software a la medida y contaba con 15 empleados, en el año 2010 cambio el giro de la empresa a consultoría de mejora de procesos de software, proporcionando capacitación en TSP, PSP y servicios de cauche de TSP. En la actualidad cuenta con una plantilla de personal de 2 empleados.

- $\quad$ yymez es una empresa dedicada al desarrollo de software, desarrollo Web, soporte y seguridad. En la actualidad cuenta con una plantilla de personal de 16 empleados. 


\subsection{Encuesta guía}

La encuesta utilizada como guía durante las entrevistas a las empresas de Zacatecas se lista a continuación:
a) ¿Qué servicios ofrece la organización?
b) ¿Con cuántos empleados cuenta la organización?
c) ¿Cuántos proyectos gestionan por año? (o-3meses, 3-6 meses, 6-9 meses o 9- 12 meses)
d) ¿Tiene roles establecidos?
e) ¿Cuántos roles puede asignarse a cada persona?
f)¿La organización cuenta con procesos? SI (1) ¿Cuál considera que es la principal motivación para implementar procesos?; (2) ¿Cuál es su experiencia en el uso de procesos? ; NO (1) ¿Cuál considera que es la principal barrera por la que su empresa no implementa procesos?

\subsection{Metodologías Agiles más utilizados}

Para la implementación de mejora de procesos de software se han identificado que las pymes han utilizado una combinación de prácticas ágiles, sin embargo han recibidlo certificaciones en tecnología y en modelos tales como Scrum, PHP, Pruebas, PSP y TSP.

En la Figura 5 muestra que el $57 \%$ utilizan una combinación de prácticas de metodologías agiles tales como Scrum, XP, Crystal, y el 43\% desarrollan de forma artesanal.

En la Figura 6 muestra que el $67 \%$ cuentan con certificaciones en tecnologías y modelos y el $38 \%$ no cuentan con certificaciones que los ayuden a mejorar sus procesos.

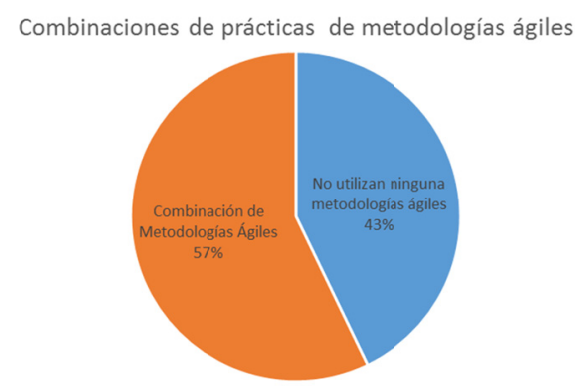

Figura 5 - Combinación de prácticas de metodologías agiles

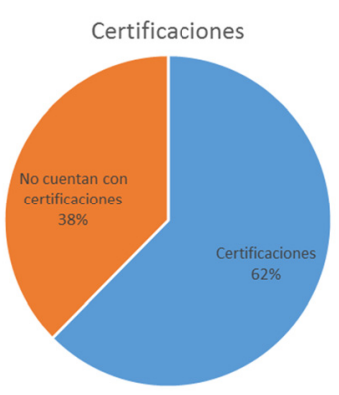

Figura 6 - Certificaciones

\subsection{Procesos mejorados}

Las prácticas antes mencionadas han sido implementadas en las pymes para mejorar sus procesos de software. Los datos arrojados en el análisis muestran que los procesos que son más enfocados para la implementación de mejora: el $37 \%$ Planificación, el $27 \%$ Pruebas, el $18 \%$ Gestión de Requisitos y finalmente el 9\% es de calidad y gestión de riesgos. 


\subsection{Principales limitaciones}

En las pymes entrevistadas las principales limitaciones que presentan para implementar una mejora de procesos de software son las siguientes:

- El personal se resiste al cambio.

- Se tiene personal recién egresado de la universidad o becados.

- No cuenta con el presupuesto para la implementación de SPI.

- No cuenta con personal capacitado para la implementación de SPI.

- No se tiene la iniciativa de implementar SPI.

- La administración de la empresa no apoya una iniciativa de SPI.

\subsection{Ventajas al implementar mejoras en las pymes}

En las pymes entrevistadas las ventajas que presentan las pymes para implementar una mejora de procesos de software (Ver Tabla 6):

Tabla 6 -Ventajas al implementar mejoras en las pymes Entrevistadas

\begin{tabular}{llc}
\hline Ventajas para la organización & \multicolumn{2}{c}{ Ventajas para los recursos humanos } \\
\hline$-\quad$ Reducción de densidad de defectos. & - & Involucrar a todo el personal de la pyme, \\
- & Involucrar al cliente, ya que se realizan & ya que se realizan reuniones diarias. \\
$\quad$ reuniones frecuentes. & - & Documentación del proceso de software. \\
$-\quad$ Mejora la calidad del producto a entregar. & - & Motivar a los empleados para la obtención \\
$-\quad$ Entregas de avances periódicamente. & & de resultados favorables. \\
& - & Reducción a la resistencia al cambio. \\
\hline
\end{tabular}

\section{Comparativa de Resultados Obtenidos}

En esta sección se muestra una comparativa de las características que fueron encontradas con la realización de la revisión literaria y las características que se encontraron mediante la investigación de campo realizada a un conjunto de pymes de la región de Zacatecas. Esta comparativa tiene como fin lograr una caracterización de las necesidades de las pymes para la implementación de mejoras.

\subsection{Comparativa de caracterización}

Esta sección muestra la caracterización realizada basada en las limitaciones identificadas en la sección 4.4 para analizar los resultados obtenidos de la revisión de literatura y los resultados obtenidos de la extracción de la información de la encuesta aplicada a pymes de la región de Zacatecas. Esta caracterización permite identificar los problemas específicos de las pymes, haciendo posible el entendimiento del entorno y necesidades de las Pymes para implementar mejoras y, por lo tanto, proporcionando información que habilite a la organización a seleccionar la mejor forma de implementar una mejora de procesos.

La Tabla 7 muestra la comparativa entre las características identificadas en las empresas analizadas de la revisión sistemática y pymes de la región de Zacatecas. 
Tabla 7 -Pymes Zacatecanas

\begin{tabular}{|c|c|c|c|}
\hline & Limitaciones & Revisión literaria & $\begin{array}{l}\text { Empresas de la } \\
\text { región de Zacatecas }\end{array}$ \\
\hline \multirow{4}{*}{ 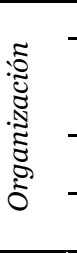 } & Son altamente dependientes de los clientes & $\mathrm{X}$ & $\mathrm{X}$ \\
\hline & $\begin{array}{l}\text { Desconocen la importancia que tiene el proceso } \\
\text { de desarrollo de software sobre la calidad del } \\
\text { producto. }\end{array}$ & $\mathrm{X}$ & $\mathrm{X}$ \\
\hline & No siguen un ciclo de desarrollo de software. & $\mathrm{X}$ & $\begin{array}{c}\text { Siguen el ciclo de vida } \\
\text { de iteración }\end{array}$ \\
\hline & Observaciones: & $\begin{array}{c}\text { Cuentan con un } \\
\text { esquema jerárquico }\end{array}$ & $\begin{array}{c}\text { Cuentan con un } \\
\text { esquema jerárquico }\end{array}$ \\
\hline \multirow{2}{*}{ 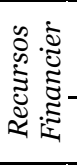 } & $\begin{array}{l}\text { Recursos limitados, no cuentan con suficiente } \\
\text { capital económico para invertir en mejora de } \\
\text { procesos. }\end{array}$ & $x_{1}$ & $x_{1}$ \\
\hline & $\begin{array}{l}\text { Dependen de apoyo externo para implementar } \\
\text { una mejora de procesos. }\end{array}$ & $\mathrm{X}$ & $\mathrm{X}$ \\
\hline \multirow{4}{*}{ 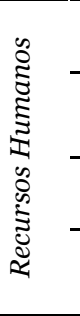 } & $\begin{array}{l}\text { Carecen de personal, el número de sus } \\
\text { empleados suelen ser mínimo. }\end{array}$ & $\mathrm{X}$ & $\mathrm{X}$ \\
\hline & $\begin{array}{l}\text { No hay roles definidos, los empleados realizan } \\
\text { varias funciones. }\end{array}$ & $\mathrm{X}$ & $\begin{array}{l}\text { Se tiene los roles } \\
\text { definidos, según sus } \\
\text { habilidades. }\end{array}$ \\
\hline & $\begin{array}{l}\text { Los empleados carecen de conocimientos sobre } \\
\text { los métodos para la mejora de procesos. }\end{array}$ & $\mathrm{X}$ & $\mathrm{X}$ \\
\hline & Observaciones & $\begin{array}{c}\text { Falta de } \\
\text { comunicación entre } \\
\text { los empleados. }\end{array}$ & $\begin{array}{l}\text { Personal sin } \\
\text { experiencia laboral. }\end{array}$ \\
\hline \multirow{3}{*}{ 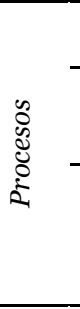 } & $\begin{array}{l}\text { No tienen procesos definidos, el software se } \\
\text { desarrolla de forma artesanal. }\end{array}$ & 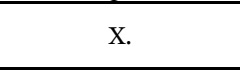 & $\mathrm{X}$ \\
\hline & $\begin{array}{l}\text { Es muy costoso implementar procesos y arrojar } \\
\text { resultados sobre la implementación de mejora } \\
\text { de proceso. }\end{array}$ & $\mathrm{X}$ & $\begin{array}{l}\text { Cuentan con prácticas } \\
\text { de Metodologías Agiles }\end{array}$ \\
\hline & $\begin{array}{l}\text { Es muy difícil adoptar un Modelo y Estándar } \\
\text { para la mejora de procesos para atacar los } \\
\text { objetivos y la visión de ésta }\end{array}$ & $\mathrm{X}$ & $\begin{array}{c}\text { Implementan una } \\
\text { mejora de procesos } \\
\text { para definir los } \\
\text { objetivos y la visión de } \\
\text { la pyme. }\end{array}$ \\
\hline \multirow{3}{*}{ 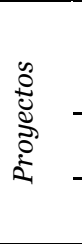 } & $\begin{array}{l}\text { Tienden a trabajar proyectos muy pequeños, los } \\
\text { cuales conllevan poco tiempo para su } \\
\text { realización. }\end{array}$ & $\mathrm{X}$ & $\begin{array}{c}\text { Proyectos entre 1-3 } \\
\text { meses de desarrollo } \\
\text { (algunos hasta dos } \\
\text { semanas). }\end{array}$ \\
\hline & $\begin{array}{l}\text { La implementación de un proceso toma mucho } \\
\text { tiempo. }\end{array}$ & $\mathrm{X}$ & $\mathrm{X}$ \\
\hline & $\begin{array}{l}\text { Implementan una mejora de procesos por que } \\
\text { los clientes lo exigen. }\end{array}$ & $\mathrm{X}$ & $\mathrm{X}$ \\
\hline \multirow{3}{*}{ 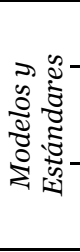 } & $\begin{array}{l}\text { Se tienen que adecuar al modelo y estándar que } \\
\text { se va a aplicar. }\end{array}$ & $\begin{array}{l}\text { No utilizan ningún } \\
\text { modelo o estándar }\end{array}$ & $\begin{array}{l}\text { No utilizan ningún } \\
\text { modelo o estándar }\end{array}$ \\
\hline & $\begin{array}{l}\text { Tienen poca o ninguna experiencia en la } \\
\text { adopción de modelos y estándares de SPI y } \\
\text { métodos de evaluación. }\end{array}$ & 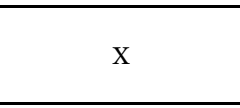 & 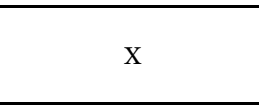 \\
\hline & $\begin{array}{l}\text { Se tienen que adecuar al modelo y/o estándar } \\
\text { que se va a aplicar }\end{array}$ & $\mathrm{X}$ & $\begin{array}{c}\text { Adoptan buenas } \\
\text { prácticas que se } \\
\text { adopten a la empresa. }\end{array}$ \\
\hline
\end{tabular}

Analizando las caracterizaciones obtenidas con la realización de la revisión de literatura y las entrevistas realizadas en pymes de la región de Zacatecanas, se han identificado las siguientes similitudes y diferencias: 
Tabla 8 -Ventajas al implementar mejoras en las pymes

\begin{tabular}{|c|c|}
\hline Similitudes & $\begin{array}{c}\text { Diferencias de las empresas } \\
\text { Zacatecanas }\end{array}$ \\
\hline $\begin{array}{l}\text { - } \quad \text { Tienen un esquema jerárquico. } \\
\text { Tienen recursos limitados, ya que no cuentan con } \\
\text { suficiente capital económico para invertir en } \\
\text { mejora de procesos. } \\
\text { Carecen de personal, ya que el número de sus } \\
\text { empleados suelen ser pequeños. } \\
\text { Los empleados carecen de conocimientos sobre los } \\
\text { métodos para la mejora de procesos. } \\
\text { No tienen procesos definidos, por lo que se } \\
\text { desarrolla el software de forma artesanal. } \\
\text { Tienden a trabajar en proyectos muy pequeños, } \\
\text { los cuales conllevan poco tiempo para su } \\
\text { realización. } \\
\text { No utilizan ningún modelo y estándar de calidad o } \\
\text { de procesos. } \\
\text { No cuenta con experiencia en la adopción de un } \\
\text { modelo y estándar de mejora de procesos. }\end{array}$ & $\begin{array}{ll} & \text { Cuentan con prácticas de metodologías } \\
\text { - } & \text { agiles. } \\
\text { Se tiene los roles definidos, según sus } \\
\text { - } & \text { habilidades. } \\
\text { - } & \text { Dependen de un sólo cliente. } \\
\text { - } & \text { Personal ciclo de vida de iteración. } \\
\text { - } & \text { Adoptan buenas prácticas que se } \\
\text { - } & \text { adopten a la empresa. } \\
\text { Implementan una mejora de procesos } \\
\text { para definir los objetivos y la visión de } \\
\text { la pyme. }\end{array}$ \\
\hline
\end{tabular}

\subsection{Comparativa de necesidades}

La Tabla 9 muestra la comparativa entre las necesidades identificadas en las empresas analizadas de la revisión sistemática y en pymes de la región de Zacatecanas.

\section{Conclusiones y Trabajo Futuro}

La mejora de procesos software habilita a las organizaciones en la creación de ventajas estratégicas respecto a sus competidores, desde la perspectiva de que la calidad de los productos depende directamente de la calidad de los procesos utilizados para su desarrollo. Sin embargo, no todas las organizaciones tienen el conocimiento para llevar este tipo de actividades de manera adecuada y por consiguiente no obtienen los resultados esperados. Esta problemática se hace más evidente en las pymes de desarrollo de software debido a las características específicas de las mismas. Con la realización de este artículo se ha podido comprobar al realizar el análisis de 7 empresas de desarrollo de software en Zacatecas y de 89 pymes desarrolladoras de software de diferentes países identificadas en los estudios primarios obtenidos de la revisión sistemática, se logró una caracterización base de las pymes y sus necesidades para la implementación de una mejora.

Tabla 9 - Comparativa de Necesidades

\begin{tabular}{lc}
\hline Necesidades Revisión Literaria & $\begin{array}{l}\text { Necesidades pymes de la región de } \\
\text { Zacatecas }\end{array}$ \\
\hline - Documentar el proceso de desarrollo de software. & \\
- Adaptar normas a su tamaño y nivel de madurez. & - Obtener presupuesto para dedicar y poder \\
- Involucrar a todo el personal. & asignar personal a la mejora de procesos. \\
- Mejorar la comunicación entre los empleados. & Contar con personal experto en las prácticas \\
- Establecer procesos de desarrollo de software con el & de software. \\
fin de producir y mantener productos de software. & - Contar con iniciativas para implementar \\
- Observar resultados tangibles en poco tiempo. & SPI. \\
- Desarrollar de un modelo que sea eficaz y que & - Contar con experiencia para implementar \\
produzca resultados favorables. & SPI. \\
\hline
\end{tabular}




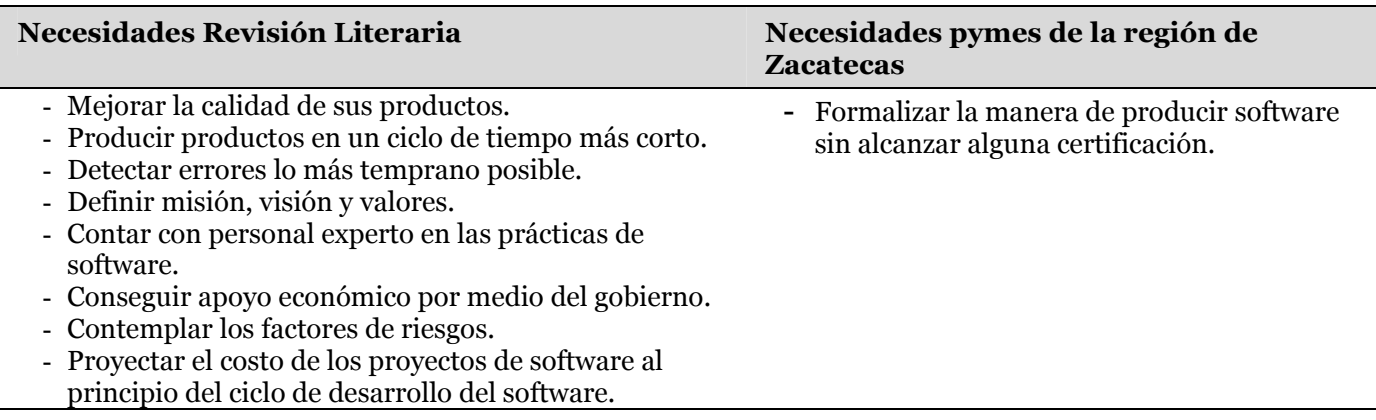

Entre los principales hallazgos encontrados se puede mencionar como principales diferencias las siguientes: (1) las pymes desarrolladoras de software entrevistadas, aún no implementan ningún modelo y estándar que les ayude a mejorar sus procesos de desarrollo; (2) las pymes entrevistadas no han implementado SPI, por tanto se concluye que esta es una característica importante que diferencia a las pymes analizadas en este artículo y; (3) la mayoría de las pymes zacatecanas tienden a tener roles definidos, en cambio las 89 pymes investigadas no los tienen hasta que se logra implementar SPI.

Basándonos en los resultados obtenidos se ha identificado como acción para apoyar a las pymes en la implementación de mejora de procesos de acuerdo a las necesidades identificadas la incorporación de herramientas que soporten y proporcionen apoyo a las pymes facilitando la implementación de mejoras en sus procesos, esto soportado por la realización talleres enfocados en el desarrollo de iniciativas de mejora de procesos, con el fin de motivar a los empleados a implementar una mejora de procesos de software.

Para cubrir esta necesidad como trabajo a futuro se está desarrollando una propuesta cuyo objetivo es identificar y definir patrones de mejora de procesos, de tal manera que se proporcione apoyo a las organizaciones en la identificación de su escenario actual, y por lo consiguiente, se les brinde ayuda dirigiendo el esfuerzo para la implementación de mejora de procesos hacía el mejor camino de acuerdo a sus características específicas. Además se desarrollará la herramienta software que soporte el uso de los patrones.

\section{Referencias Bibliográficas}

Bedini, G. A., Llamosa, A., Pavlovic, M., \& Steembecker, K. (2005) "Quality Software Map of South America." Proceedings of the First International Research Workshop for Process Improvement in Small Settings, 216-227.

Calvo-Manzano, J. A., Cuevas, G., San Feliu, T., De Amescua A., García, L., \& Pérez, M. (2002). Experiences in the application of software process improvement in SMES. Software Quality Journal, 10(3), 261-273.

Hogan, J. M., Smith, G., and Thomas, R. (2002). "The Real World Software Process." Proceedings of the Ninth Asia-Pacific Software Engineering Conference, 366- 375. 
Ibrahim, S., \& Ali, R. Z. R. M. (2011). Study on acceptance of customised Software Process Improvement (SPI) model for Malaysia's SME. 2011 Malaysian Conference in Software Engineering, 25-30.

Jeners, S., Clarke, P., \& O’Connor, R. (2013). Harmonizing Software Development Processes with Software Development Settings-A Systematic Approach. Systems, Software and Services Process Improvement, 364, 167-178.

Kitchenham, B. \& Charters, S. (2007). Guidelines for performing systematic literature reviews in software engineering. Technical Report EBSE 2007-001, Keele University and Durham University Joint Report.

Landaeta, J., \& Amescua, A. (2008). "Práctico SPI Planificación." EuroSPI 2008, 8293.

Mas, A., \& Amengual, E. (2005). "La mejora de los procesos de software en las pequeñas y medianas empresas (pyme). Un nuevo modelo y su aplicación a un caso real " REICIS Revista Española de Innovación, Calidad e Ingeniería del Software, 7-29.

Ministerio de Industria, Energía y Turismo. (2013) "Más Información sobre la nueva definición de la PYME en UE",disponible en :http://www.ipyme.org/esES/CreacionEmpresas/Documents/NuevaDefinicionPyme.pdf

Moreno T.M. (2008). "Cuatro talones de Aquiles de las pymes”, Observatorio PYME artículos en línea, disponible:http://www.observatoriopyme.org/index.php?option=com_content\&vi ew=article\&id=74\&Itemid $=102$.

Muñoz, M., Mejia, J., Calvo-Manzano, J.A., Cuevas, G., San Feliu, T., \& De Amescua, A. (2012). "Expected Requirements in Support Tools for Software Process Improvement in SMEs." Electronics, Robotics and Automotive Mechanics Conference (CERMA),135 - 140.

Pino, F. J., García, F., \& Piattini, M. (2007). Software process improvement in small and medium software enterprises: a systematic review. Software Quality Journal, 16(2), 237-261.

Pino, F., García, F., \& Piattini, M. (2009). An integrated framework to guide software process improvement in small organizations. Software Process Improvement, 23(122), 213-224.

Revankar, A., Mithare, R., \& Nallagonda, V., (2005). "Accelerated Process Improvements for Small Settings”, Proceedings of the First International Research Workshop for Process Improvement in Small Settings,117-126.

Scott, L., Jeffery, R., Carvalho, L., D’Ambra, J., \& Rutherford, P. (2001). Practical software process improvement - the IMPACT project. Proceedings 2001 Australian Software Engineering Conference, 182-189. 
Secretaria de Economía, Pymes, (2013) "PyMES, eslabón fundamental para el crecimiento en México" Disponible en: http://www.promexico.gob.mx/negociosinternacionales/pymes-eslabon-fundamental-para-el-crecimiento-enmexico.html.

Serrano, M. a., de Oca, C. M., \& Cedillo, K. (2003). An experience on using the team software process for implementing the Capability Maturity Model for software in a small organization. Third International Conference on Quality Software, 2003. Proceedings. 327-334.

Valdés, G., Astudillo, H., Visconti, M., \& López, C. (2010). The Tutelkan SPI Framework for small settings: A methodology transfer vehicle. In Systems, Software and Services Process Improvement, 142-152.

Valtierra, C., Muñoz M. \& Mejia, J. (2013). "Characterization of Software Process Improvement Needs in SMEs". International Conference on Mechatronics, Electronics and Automative Engineering (ICMEAE), 223-234. 